\title{
Laparoscopic Removal of Local Renal Cell Carcinoma Recurrence
}

\author{
Alexander Tsivian, Shalva Benjamin, Avraham Shtricker, Matvey Tsivian, Shlomo Kyzer, \\ A. Ami Sidi \\ Departments of Urologic Surgery (SK) and Surgery B (AT, SB, AS, MT, AAS), Wolfson Medical Center, \\ Holon, Israel
}

\begin{abstract}
Purpose: To describe an entirely laparoscopic technique for excising a recurrence of local renal cell carcinoma (RCC). Materials and Methods: The patient is placed in a full flank position. A 10-mm trocar is inserted using Hasson's technique with three additional ports in the upper abdomen. After lysis of adhesions, the psoas muscle, ureteral and gonadal vein remnants, inferior vena cava or aorta, and renal vessel stumps are dissected and isolated. The specimen, including the mass, the adrenal gland, and the ipsilateral pararenal and paracaval or para-aortic tissue within Gerota's fascia remnants, are excised en bloc and removed inside an Endocatch-II bag.

Results: To date we have used this technique for excising RCC recurrences in three patients. Pathologic examination showed clear cell type RCC Fuhrman grade 2 in the specimens of two patients and chromophobe type in one. No patient have had further recurrence after 50, 38 and 12 months of follow-up.

Conclusions: An entirely laparoscopic surgical approach for excising local RCC recurrence has not, to our knowledge, been previously described. This method can be effectively applied while adhering to oncologic principles, with minimal blood loss and low morbidity.
\end{abstract}

Key words: carcinoma, renal cell; recurrence; laparoscopy

Int Braz J Urol. 2009; 35: 436-41

\section{INTRODUCTION}

Local recurrence of renal cell carcinoma (RCC) is rare (1-3) and surgical treatment represents an effective therapeutic option in these cases. As experience in minimally invasive surgery grows, laparoscopic procedures become viable alternatives to conventional open surgery.

To our knowledge, we report the first case series of a purely laparoscopic technique for excising local RCC recurrence.

\section{MATERIALS AND METHODS}

We retrospectively reviewed medical records of patients with solitary kidney bed recur- rence of RCC, treated with a purely laparoscopic technique. We obtained a brief clinical summary of these cases, present the surgical technique and have delineated the possible pitfalls in using this procedure.

\section{Patients}

Case 1: a 60-year-old male underwent an open retroperitoneal removal of a 7-cm mass in the renal fossa after having undergone a retroperitoneal left radical nephrectomy 4 years earlier. Two years later, the patient required surgery for removal of a $2-\mathrm{cm}$ tumor from the renal fossa, and this time it was carried out entirely by a laparoscopic transperitoneal approach. An inadvertent descending colon injury was identified and repaired intraoperatively. 
Case 2: a 65-year-old male had undergone a lumbar right nephrectomy for T1N0M0 RCC 7 years earlier. A follow-up magnetic resonance imaging (MRI) evaluation revealed a $5-\mathrm{cm}$ mass in the kidney bed (Figure-1).

Case 3: a 73-year-old male underwent an open transperitoneal right nephrectomy for T1N0M0 RCC 6 years earlier. A 4-cm local recurrence was later identified.

\section{Surgical Technique}

The surgical technique has been previously reported (4). The patient is placed in a full flank position. Four ports are used: one $10-\mathrm{mm}$ trocar is placed using Hasson's technique supraumbilically, one 5-mm port is placed in the midclavicular line in the ipsilateral upper quadrant, and one 12-mm trocar is placed above the first one in the midline between the xyphoid and the first port. The fourth 5-mm trocar is inserted above the former trocar on the right side and in the anterior axillary line on the left side (Figure-2). After lysis of adhesions, the psoas muscle, ureteral and gonadal vein remnants, inferior vena cava or aorta, and renal vessels stumps are dissected and isolated. Harmonic scalpel ${ }^{\mathrm{TM}}$ (Ethicon, Cincinnati, $\mathrm{OH}, \mathrm{USA}$ ) is employed during the dissection. The specimen, including the mass, the adrenal gland, and

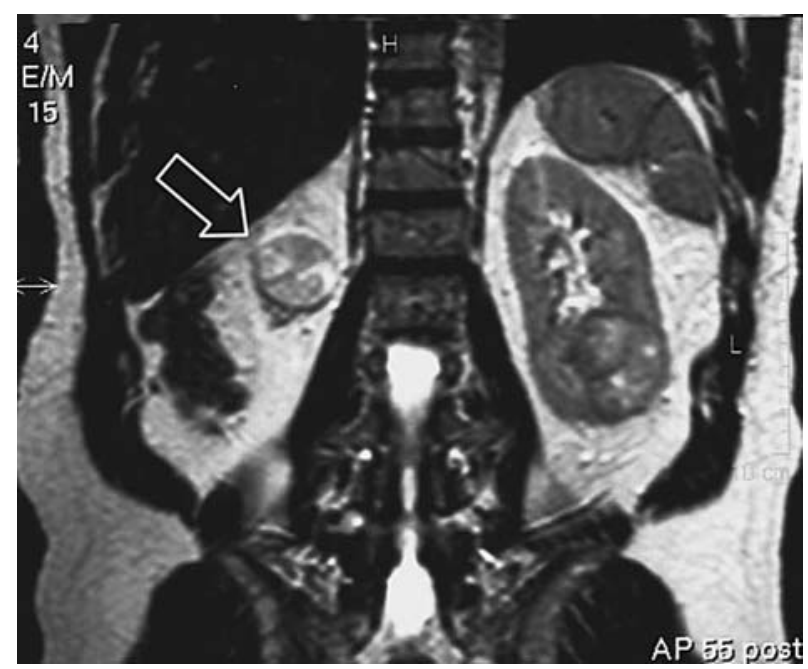

Figure 1 - Magnetic resonance imaging. We can see a 5-cm mass in the kidney bed (arrow), and a $5 \mathrm{~cm}$ mass in a single left kidney.

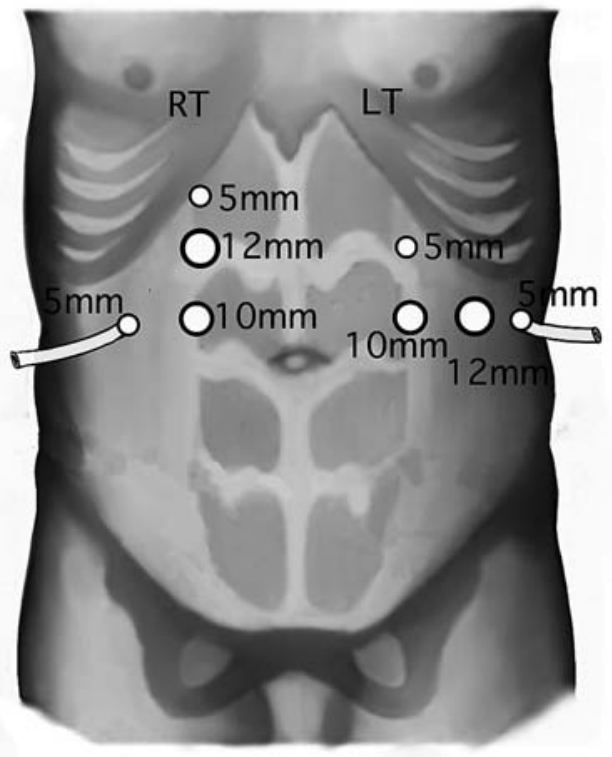

Figure 2 - Sites for proposed trocars placement.

the pararenal and paracaval (para-aortic) tissue within Gerota's fascia remnants (Figure-3), is excised en bloc, placed in an Endo Catch-II (Covidien, Boston, MA, USA) bag and removed through an additional

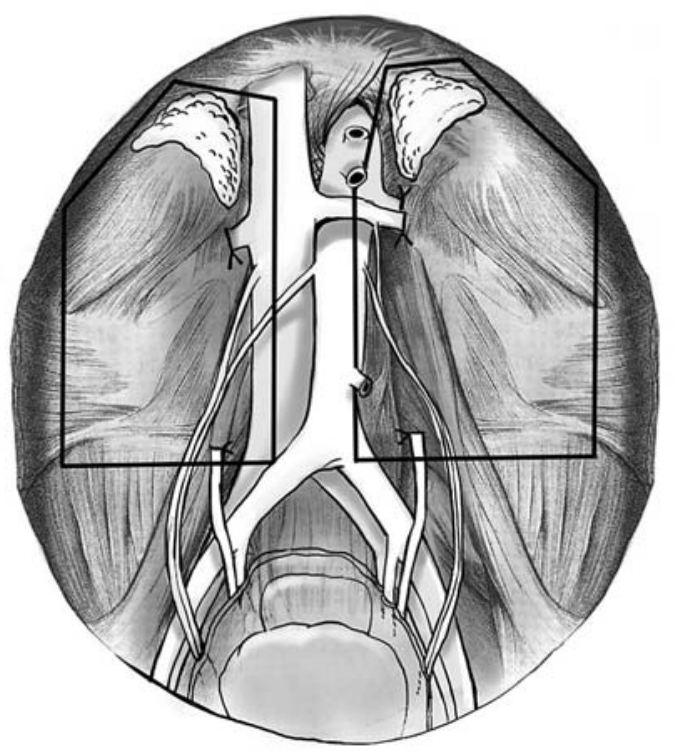

Figure 3 - The proposed templates for right and left retroperitoneal tissue dissection for a solitary local recurrence of renal cell carcinoma. 
6-cm incision in the ipsilateral lower quadrant of the abdomen.

\section{RESULTS}

To date, 3 patients with solitary local recurrences have been treated with purely laparoscopic technique at our center.

A post-lumbotomy ventral hernia that was identified in case 2 was repaired laparoscopically concomitantly with an open partial nephrectomy for a $5-\mathrm{cm}$ tumor in the left (contralateral) kidney. Operative times were between 110-150 min. Blood loss ranged between 30-200 mL. No blood transfusions were required peri- or postoperatively and the postoperative course was uneventful. Hospital stay was 2, 6 and 7 days in our patients.

Pathology revealed clear cell RCC Fuhrman grade 2 in two patients and chromophobe type RCC in one. With follow-up of 12-50 months, there was no evidence of recurrence.

\section{COMMENTS}

Isolated local $\mathrm{RCC}$ recurrence after radical nephrectomy is reported to occur in $2 \%$ of patients $(5,6)$. There are scarce reported data on the incidence, presentation and therapy of isolated local RCC recurrence, and the therapeutic options are surgery alone or surgery combined with adjunctive therapies (radiotherapy and chemo-/immunotherapy) as well as non-surgical modalities (3). Oncological outcomes of the proposed treatment options differ largely. Itano et al. (1) concluded that long-term survival with locally recurrent $\mathrm{RCC}$ is poor, with a $28 \%$ survival rate at 5 years. However, patients treated with radical surgical resection of the recurrence had a 5-year cause-specific survival rate of $51 \%$, whereas adjuvant medical treatment alone yielded $18 \%$, similar to the $13 \%$ of patients on observation alone. In their retrospective study of 15 patients with local recurrence, Weisner et al. (3) reported a cancer-specific survival (CSS) rate of $47 \%$ at 1 year and $33 \%$ at 3 years in surgically treated patients. The mean CSS was 62.2 months (38-110 months) after surgery, 26 months (8-74 months) after resection and adjunctive treatment, and 9.2 months (7-15 months) after chemo-immunotherapy.

In our opinion, and in accordance with the literature, isolated renal fossa recurrences should be approached surgically whenever technically feasible. In most cases, surgical extirpation of the solitary recurrent tumor may be possible and, if accompanied by tumor clearance, could lead to prolonged distant and local disease-free survival (2).

The reported time to further tumor progression (TTP) was 13.9 months (0-32 months) for patients with isolated local recurrence and 4.2 months (3-9 months) for patients with synchronous metastasis (3). The mean TTP after surgery alone was 22.2 months (11-32 months) (3). In fact, with one exception, all reported series of resection of RCC local recurrence (1-3,5-7) involve open surgical techniques. For example, Sandhu et al. (2) used a thoraco-abdominal incision with removal of the eighth rib in 14 out of 16 patients: they noted that the approach gives excellent exposure but is traumatic for the patient. A complete resection may be technically challenging (8). Along with reducing surgical trauma, laparoscopy has several other advantages over conventional open surgery. It magnifies the anatomy, allowing clear visibility of details by the entire operating team, as well as providing the opportunity to record these details for teaching purposes.

Our literature search revealed that Nakada et al. (9) reported a case of local RCC recurrence excision using a hand-assisted laparoscopic technique. The same group presented a series of 5 cases treated with the same approach (10). Although not a pure laparoscopic technique, the authors commented that this approach minimizes postoperative morbidity and offers the ability to palpate the lesion in the renal fossa, thereby simplifying identification and resection of the mass. It is reasonable to propose that pure laparoscopy could further enhance the advantages of minimally invasive surgery. Berger et al. (11) recently reported a successful case of solitary inter-aorto-caval RCC metastasis resection with a purely laparoscopic technique. They concluded that laparoscopic approach is feasible in experienced hands and may confer the advantage of a faster recovery, compared to open surgical management. In our series, hospital stay was 2, 6 and 7 days in our patients. These data should be 
considered in view of a colon injury in one patient (6 days) and multiple surgical procedures, including a contralateral open partial nephrectomy in another (7 days).

Several controversial surgical technique points should be discussed. We believe that careful planning of the surgical dissection planes and defining the borders of the tissue packet to be removed to avoid tumor manipulation (whenever possible), are the keys to a successful oncologic laparoscopy. For recurrent RCC, we believe it is necessary to remove all retroperitoneal fat tissue and the ipsilateral adrenal (if left in situ during prior radical nephrectomy), and not merely the presenting lesion. Therefore, this surgery should only be carried out by experienced surgeons in strict adherence to oncologic principles.

To date, there are no widely accepted standards or rules for retroperitoneal dissection. For example, it remains unclear whether a full retroperitoneal lymph node dissection should be performed in all cases or a limited dissection may, in fact, be sufficient. Based on our experience we suggest limited lymph node dissection (Figure-3) as adequate treatment in cases of solitary local recurrence of RCC. According to the template, dissection of retroperitoneal tissue should extend from the level of diaphragmatic crura down to great vessel bifurcation, including vena cava dissection with paracaval lymph nodes for right sided tumors, and, for left-sided recurrences - dissection of aorta with para-aortic nodes. Inter-aorto-caval lymph nodes were not dissected.

Regarding possible indications for laparoscopic excision of local RCC recurrence, we suggest solitary kidney bed masses with clearly identifiable borders and the possibility to visualize on preoperative imaging cleavage planes between the mass and adjacent organs that may enable planning radical extirpation of the tumor. Whenever adjacent organs are involved and no cleavage plane can be seen on imaging, or in presence of multiple masses, choosing laparoscopic intervention may not be prudent. comfortably

Correct trocar placement is essential for a performed procedure and minimal limitations of visual field and instrument maneuverability. In establishing instrument ports, we plan the classical triangulation referring to the former renal hilum as an anchor point for the optics channel. Working ports are placed accordingly (Figure-2).

Once peritoneal cavity is accessed, dissection template is visually delineated identifying the appropriate anatomical hallmarks (Figure-3). Psoas muscle represents the deep border of dissection whereas the great vessels (aorta and vena cava in left- and rightsided procedures, respectively) define the medial limits of the template. These structures are carefully dissected forming the en bloc excision packet containing the tumor. By using this approach, oncological principles are preserved and tumor manipulation is minimized. We do not use mechanical suturing in the former renal hilum area. Metallic or plastic clips placed during previous surgery may represent harsh terrain for EndoGIA ${ }^{\mathrm{TM}}$ stapling and cause inefficient clipping or "malfunction" of the instrument. Using instruments like the Harmonic scalpel and/or LigaSure $^{\mathrm{TM}}$ vessel sealing system (Valleylab, CO, USA) have been useful during dissection.

Operating on a "surgically violated" site may be challenging in open surgery and is moreover of major concern when approaching these cases laparoscopically due to adhesions. Accurate patient selection is paramount in these cases. Moreover, advanced laparoscopic expertise is required to safely perform these complex procedures. In our series, a colon tear occurred during a difficult dissection and repaired with EndoGIA ${ }^{\text {TM }}$. Surgeons should be ready to promptly identify and repair inadvertent injuries as in this case.

In our series, oncological outcomes remain encouraging. However, these results should be interpreted in light of highly selected patients - a solitary recurrence involving the kidney bed years after initial treatment with no evidence of further involvement. These patients may represent the ideal candidates for surgical intervention. An obvious limitation of our report is its sample size and results should be interpreted accordingly. Larger series would allow for more conclusions. Moreover, our follow-up does not go beyond 5 years, therefore long-term outcomes remain unclear.

In conclusion, we report encouraging results of a purely laparoscopic resection of solitary renal fossa recurrences following a radical nephrectomy, strictly adhering to oncologic principles. 


\section{ACKNOWLEDGMENT}

We thank Janice M Mayes for her valuable assistance in preparation of this manuscript.

Presented in part at European Association of Urology Congress, 2006, Paris, France.

\section{CONFLICT OF INTEREST}

None declared.

\section{REFERENCES}

1. Itano NB, Blute ML, Spotts B, Zincke H: Outcome of isolated renal cell carcinoma fossa recurrence after nephrectomy. J Urol. 2000; 164: 322-5.

2. Sandhu SS, Symes A, A'Hern R, Sohaib SA, Eisen T, Gore M, et al.: Surgical excision of isolated renal-bed recurrence after radical nephrectomy for renal cell carcinoma. BJU Int. 2005; 95: 522-5.

3. Wiesner C, Jakse G, Rohde D: Therapy of local recurrence of renal cell carcinoma. Oncol Rep. 2002; 9: 189-92.

4. Tsivian A, Benjamin S, Kheifets A, Sidi AA.Laparoscopic removal of local recurrence of renal cell carcinoma ( RCC ). Eur Urol. 2006; 5 (Suppl): 300. Abstract \# V18.
5. Tanguay S, Pisters LL, Lawrence DD, Dinney CP: Therapy of locally recurrent renal cell carcinoma after nephrectomy. J Urol. 1996; 155: 26-9.

6. Esrig D, Ahlering TE, Lieskovsky G, Skinner DG: Experience with fossa recurrence of renal cell carcinoma. J Urol. 1992; 147: 1491-4.

7. Bruno JJ 2nd, Snyder ME, Motzer RJ, Russo P: Renal cell carcinoma local recurrences: impact of surgical treatment and concomitant metastasis on survival. BJU Int. 2006; 97: 933-8.

8. Tombolini P, Ruoppolo M, Malagola G, Rovera F: Metastatic retroperitoneal renal cell carcinoma. A case for more aggressive approach. Risk and results. Arch Ital Urol Androl. 2005; 77: 129-30.

9. Nakada SY, Johnson DB, Hahnfield L, Jarrard DF: Resection of isolated fossa recurrence of renal-cell carcinoma after nephrectomy using hand-assisted laparoscopy. J Endourol. 2002; 16: 687-8.

10. Bandi G, Wen CC, Moon TD, Nakada SY: Single center preliminary experience with hand-assisted laparoscopic resection of isolated renal cell carcinoma fossa recurrences. Urology. 2008; 71: 495-9; discussion 499-500.

11. Berger A, Aron M, Canes D, Gill IS: Laparoscopic management of interaortocaval metastases of renal cell carcinoma. J Endourol. 2008; 22: 2381-4; discussion 2384.

\section{Correspondence address:}

Dr. Avraham Shtricker

Department of Urologic Surgery

The E. Wolfson Medical Center

PO Box 5

Holon, 58100 Israel

Fax: 972-3-502-81-99

E-mail: shtrickeravi@hotmail.com 


\section{EDITORIAL COMMENT}

About $20 \%$ to $30 \%$ of patients with organconfined renal cancer will develop metastases after primary therapy (1-3). Whereas most patients with metastatic disease will develop disseminated metastases, less than $5 \%$ will be solitary, and only $1 \%$ to $2 \%$ will have an isolated renal fossa recurrence after radical nephrectomy (4).

Management of local recurrence can be challenging, with relatively few series reported in the literature. However, surgical excision of isolated renal fossa recurrences with or without adjuvant therapy yields better 5-year survival rates in contrast to systemic therapies or observation alone (5).

Since laparoscopic techniques have increasingly been gaining acceptance, and with the greater distribution of this technique, the indications for the future seems very promising.

The authors report their experience with the laparoscopic management of local recurrence of RCC in 3 cases with good results and presented their data at the EAU-meeting. In renal cell cancer, surgery of single metastasis is crucial, because systemic therapy remains palliative with limited long-term results. If successful surgery can delay the onset of systemic therapy, survival can possibly be prolonged with a longer symptom free interval for selected cases (5). A minimal approach in patients, who will most likely require additional procedures and / or systemic therapy in the near future, is beneficial under these circumstances.

Of course, there is no "standard surgery" in local recurrence of kidney cancer, there will always be pro's and con's for any kind of management. There exists limited data in the literature regarding minimal invasive treatment of local recurrence or metastasis, and the paper of Tsivian et al. is the first case report of pure laparoscopic management. The authors have pointed out the specific problem in this issue and propose this procedure as suitable for highly selected patients.

And this fact should be considered: Due to the higher degree of experience, the indication for laparoscopic surgery will be different in specialized centers as in institutions with a lower case number. If single site metastases seem to be suitable for a minimal invasive approach laparoscopy is always worth an attempt because, the benefit for the patient is significant whereas safety and outcome is as good as in open surgery if performed in a specialized center.

\section{REFERENCES}

1. Dekernion JB, Ramming KP, Smith RB: The natural history of metastatic renal cell carcinoma: a computer analysis. J Urol. 1978; 120: 148-52.

2. Kuczyk MA, Anastasiadis AG, Zimmermann R, Merseburger AS, Corvin S, Stenzl A: Current aspects of the surgical management of organ-confined, metastatic, and recurrent renal cell cancer. BJU Int. 2005; 96: 721-7.

3. Sandock DS, Seftel AD, Resnick MI: Adrenal metastases from renal cell carcinoma: role of ipsilateral adrenalectomy and definition of stage. Urology. 1997; 49: 28-31.

4. Schrodter S, Hakenberg OW, Manseck A, Leike S, Wirth MP: Outcome of surgical treatment of isolated local recurrence after radical nephrectomy for renal cell carcinoma. J Urol. 2002; 167: 1630-3.

5. Master VA, Gottschalk AR, Kane C, Carroll PR: Management of isolated renal fossa recurrence following radical nephrectomy. J Urol. 2005; 174: 473-7; discussion 477.
Dr. Andreas H. Wille Department of Urology University Hospital Charité Humboldt-University Berlin Berlin, Germany E-mail:andreas.wille@charite.de 